\title{
Chemokines and cytokines network in the pathogenesis of the inflammatory skin diseases: atopic dermatitis, psoriasis and skin mastocytosis
}

\author{
Bogusław Nedoszytko', Małgorzata Sokołowska-Wojdyłoº', Katarzyna Ruckemann-Dziurdzińska², \\ Jadwiga Roszkiewicz ${ }^{1}$, Roman J. Nowicki ${ }^{1}$
}

${ }^{1}$ Department of Dermatology, Venereology and Allergology, Medical University of Gdansk, Poland Head of Department: Prof. Roman J. Nowicki MD, PhD

${ }^{2}$ Department of Pathology and Experimental Rheumatology, Medical University of Gdansk, Poland

Head of Department: Prof. Ewa Bryl MD, PhD

Postep Derm Alergol 2014; XXXI, 2: 84-91 DOI: $10.5114 /$ pdia.2014.40920

\begin{abstract}
Chemokines are signaling peptides which regulate cell trafficking and provide control of the tissue-specific cell homing. In the skin, chemokines are secreted both by the resident cells such as keratinocytes, melanocytes, fibroblasts, dendritic cells and mast cells, as well as by infiltrated cells - lymphocytes, eosinophils, and monocytes. Chemokines, together with cytokines, participate in induction and maintenance of inflammation in the skin and regulate the composition of the cellular infiltrates. Inflammation within the skin is a feature shared by atopic dermatitis and psoriasis, two of the most common dermatoses. Accumulation of activated mast cells in the affected skin is seen both in atopic dermatitis and in psoriasis. This paper presents a concise overview of the current knowledge on the role chemokines have in pathogenesis of atopic dermatitis, psoriasis, and mastocytosis, a disease caused directly by the accumulation and activation of mast cells in the skin.
\end{abstract}

Key words: chemokine, cytokines, atopic dermatitis, psoriasis, mastocytosis.

\section{Introduction}

Cytokines are small, hormone-like, signal peptides produced in cells activated by the inflammatory agents. Cytokines were initially named lymphokines or monokines to emphasize their cell of origin. Today, the term "cytokines" is used because any nucleated cell can secrete them. To date, a total of more than 100 cytokines have been described with different functions and different places of origin. In the skin, cytokines are produced by resident cells, such as keratinocytes, Langerhans cells (LC), melanocytes, mast cells (MC), macrophages, and by recruited cells of the inflammatory infiltrates: neutrophils, eosinophils and lymphocytes. Cytokines are, in general, not stored in the cells, but are synthesized de novo following cell activation. Cytokines include lymphokines, monokines, interleukins, interferons, growth factors and chemokines. Cytokines, unlike blood-transported endocrine hormones, act locally within the tissue, on the neighboring cells (paracrine action) or on the producing cells themselves (autocrine action). In the case of a prolonged action of a strong inflammatory stimulus, cytokine production may become excessive. In such a case they can get into plasma and affect cells distant from the place of the original inflammation in an endocrine manner. Cytokines act through specific membrane receptors found on all cells constituting the body. Cytokine receptors can also be found in the blood in a free form. Cytokine molecule can stimulate the cell only if it binds with a cell receptor. Cytokine molecule binding to a soluble free receptor neutralizes it. The structures of the cytokine receptors are often homologous, so many cytokines have multidirectional pleiotropic effects. Some of the cytokines, in addition to their pleiotropic action, may have a synergistic effect on the same cells, and other may act antagonistically. The same cytokine may also act differently on different cells (redundancy). As a result, in each tissue, a complex and precisely regulated cytokine network is formed [1, 2].

Cytokines control all phases of the immune response: induction, effector phase and termination. They regulate both innate immunity mechanisms and specific cellular

Address for correspondence: Małgorzata Sokołowska-Wojdyło MD, PhD, Department of Dermatology, Venereology and Allergology, Medical University of Gdansk, 7 Dębinki St, 80-210 Gdansk, Poland, phone: +48 602618 195, e-mail: mwojd@gumed.edu.pl Received: 22.02.2013, accepted: 23.06.2013. 
and humoral immune responses by affecting cell proliferation, differentiation and activation of $B$ and $T$ lymphocytes as well as NK cells, monocytes/macrophages, granulocytes and keratinocytes. Cytokines influence the function and migration of mature neutrophils, mast cells, basophils and eosinophils by activation of adhesion molecules and activation of the synthesis of chemotactic factors. Cytokine cross-talk is extremely complex. Different cytokines may have an antagonistic, additive or synergistic influence on the same biological process [1, 2].

Based on their in vivo activity, cytokines can be divided into pro-inflammatory, anti-inflammatory, mitogenic (i.e. regulating cell growth and proliferation), hematopoietic and chemotactic (chemokines) ones.

Inflammation, although with a different composition of the cellular infiltrate, is a common denominator of three skin diseases: atopic dermatitis, psoriasis and cutaneous mastocytosis, which are the subject of our interest.

The aim of this paper is to present a concise overview of the role of cytokines and chemokines in the pathogenesis of the abovementioned diseases.

Among the numerous pro-inflammatory cytokines interleukins (IL) have a particularly strong representation with IL-1, IL-6, IL-8, IL-12, IL-15, IL-17 and IL-18. In addition, the following cytokines also have proinflammatory properties: tumor necrosis factor- $\alpha$ (TNF- $\alpha$ ), interferon- $\gamma$ (IFN- $\gamma$ ), oncostatin M (OSM), granulocyte-macrophage colony-stimulating factor (GM-CSF) and macrophage colony-stimulating factor (M-CSF). Proinflammatory cytokines stimulate both cellular and humoral inflammatory response - activation, adhesion and aggregation of neutrophils and mast cells, lymphocyte proliferation, antibody production in B cells, liver production of acute phase proteins and hypothalamic fever reaction. Interleukin- 1 and TNF- $\alpha$ are primary inflammatory cytokines as they are produced during the early cellular response to inflammatory stimuli (e.g. bacterial lipopolysaccharide), which activate the cell towards the production of secondary inflammatory cytokines (IL-6, IL-8, IL-12, IL-15, IL-17, IL-18). Anti-inflammatory cytokines including IL-4, IL-10, IL-11, IL-13 and TNF- $\beta$ exert their anti-inflammatory effect by inhibiting the production of pro-inflammatory mediators and by stimulation of the production of the soluble receptors to the cytokines. Growth and proliferation regulators comprise another cytokine group. IL-2, IL-4, IL-5, IL-12, PDGF, VEGF, FGF, EGF and TGF- $\beta$ stimulate cells of the immune system to proliferate and grow. Hematopoietic cytokines including GM-CSF, M-CSF, G-CSF, SCF, hemopoietin and IL- 6 stimulate the proliferation of the bone marrow stem cells. Chemotactic cytokines, for example IL-8, RANTES (regulated on activation, normal T expressed and secreted) and monocyte chemotactic protein-1 (MCP-1), determine the chemical gradient for the leukocytes to follow towards the inflammation site [2-10].
Chemokines are a group of 8-11 kDa proteins, which may be classified into four sub-groups, according to the polypeptide chain cysteine location: C, CC, CXC and, CX3C [5]. Nomenclature of chemokines and their receptors is based on the numbers allocated according to the order in which they were discovered and described. Each number is accompanied by letter $\mathrm{L}$ - for the ligand or $\mathrm{R}$ - for the receptor. Most of the known chemokines (over 50) belong to the CC and CXC families. Chemokines interact with the cells via extracellular domains of the receptor proteins. Some receptors bind multiple chemokines, and various chemokines can interact with several different receptors (Figure 1) [2-10].

Regulation of white blood cells trafficking, necessary for maintenance of the normal immune system function and for the defense against pathogens, is the primary function of the chemokines. However, chemokines are
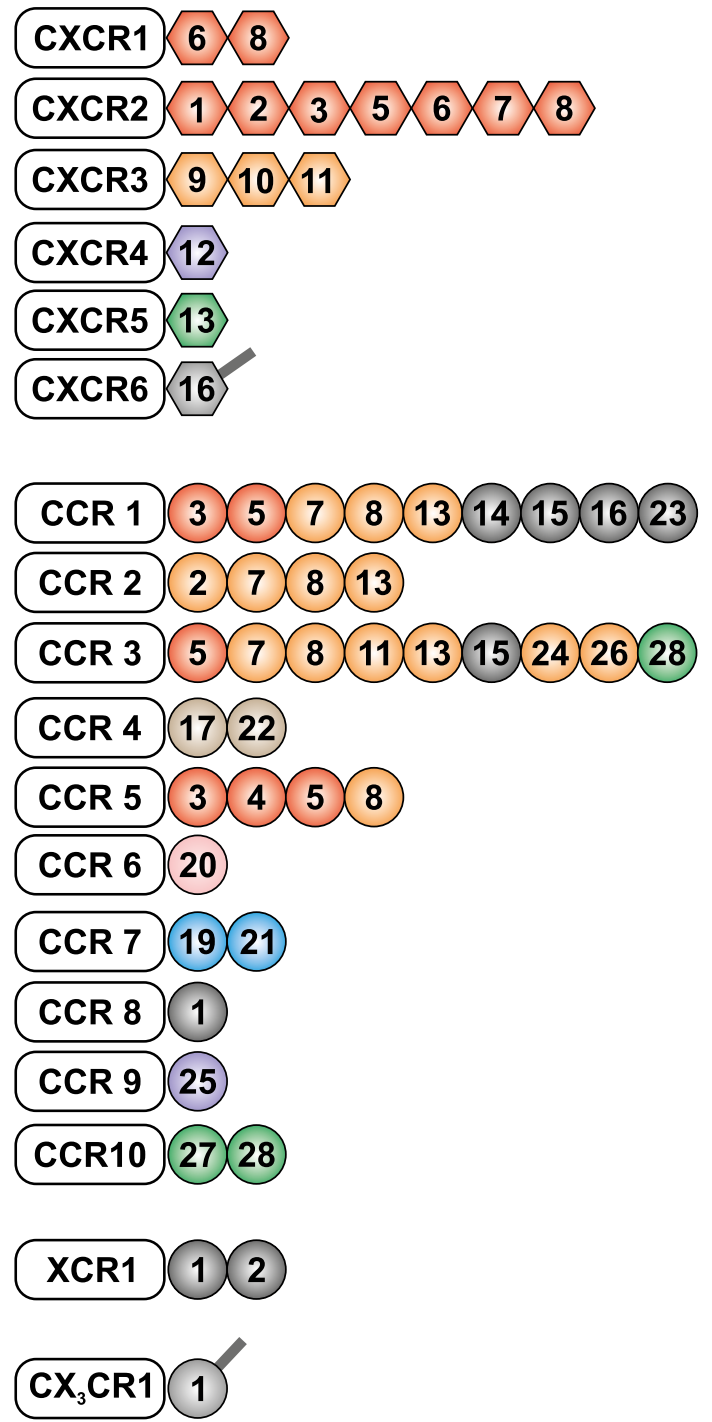

Figure 1. Chemokine receptors and their ligand chemokines [3] 
not solely chemoattractants for the immune system cells and have a wide range of effects in many different cell types beyond the immune system. It is already known that chemokines play some role in neoangiogenesis, in embryogenesis and in organogenesis. Excessive chemokine activation and synthesis is a hallmark of pathogenesis of many autoimmune (rheumatoid arthritis, psoriasis), inflammatory (allergies, asthma, atherosclerosis) and neoplastic diseases [2-10].

Chemokines can be divided into constitutive (alternatively called homeostatic, housekeeping or lymphoid) and inducible (inflammatory) ones, based on the conditions of their production. Constitutive chemokines are continuously produced in discrete microenvironments within lymphoid or non-lymphoid tissues. They are involved in maintaining physiologic traffic of lymphocytes and dendritic cells. Constitutive chemokines control the relocation and recirculation of leukocytes. They are also involved in the maturation, differentiation and activation of the lymphocytes and of the dendritic cells. CCL19 and CCL21, produced in the lymph node, are examples of constitutive chemokines. CCL19 and CCL21 induce the migration of immature dendritic cells possessing CCR7 receptor from the skin to the lymph node $[3,11]$.

Most chemokines produced in the human body are inflammatory chemokines. The MCP-1 (CCL2), RANTES (CCL5) and eotaxin (CCL11) are representatives of this group. Inflammatory chemokines are produced in an inducible manner by various tissues and infiltrating leukocytes in response to bacterial toxins and following activation by the key pro-inflammatory cytokines, such as IL-1, TNF and interferons. The main task of inflammatory chemokines is to regulate leukocyte recruitment towards the site of inflammation or infection in order to provide the host defense. Extensive literature documenting the expression of cytokines and chemokines in the tissues and body fluids, tissues collected from patients during surgical procedures, in the chronic or acute inflammation has shown that ligands for CCR1, CCR2, CCR5, and CXCR3 are ubiquitous in chronic inflammation while in acute inflammation, CXCR1 and CXCR2 binding chemokines dominate. CXCR1 and CXCR2 are receptors of the activated neutrophils and T lymphocytes; CCR5 are monocyte receptors; CCR1 and CCR2 are receptors present on monocytes, eosinophils and basophils; CXCR3 are receptors of Th1 lymphocytes [2, 3].

Chemokines are involved in many inflammatory skin diseases such as psoriasis, atopic dermatitis and mastocytosis but the understanding of their exact role in the pathogenesis of these diseases remains incomplete.

\section{Cytokines and chemokines in the pathogenesis of psoriasis}

Psoriasis is one of the most common diseases of the skin. It affects $2-3 \%$ of the European population.
The main cause underlying psoriasis remains unknown. The histological abnormalities in a psoriatic skin lesion include an excessive proliferation and impaired maturation of epidermal cells, i.e. abnormal keratinization (parakeratosis), as a result of the shortening of the epidermis renewal time (from 28 to 3-4 days) and of the expression of specific keratins. Chronic dermatitis, localized or generalized, with infiltration of the dermis and subcutaneous tissue by CD4+ T cells, neutrophils and macrophages, activation of mast cells, infiltration of cytotoxic CD8+ lymphocytes into the epidermis (Munro microabscesses), and abnormal development of blood vessels (neoangiogenesis) are characteristic features of psoriasis. Ten-thirty percent of patients develop arthritis, which can lead to a permanent disability. CD4 lymphocytes of Th1 and Th17 cytokine profile together with CD8 Tc1 lymphocytes play an essential role in the pathogenesis of psoriasis. These cells migrate to the skin, and through their activation and cytokine- and chemokine-mediated interaction with other cells of the skin and epidermis evoke the inflammation. An increased expression of the following pro-inflammatory cytokines (at RNA and protein level) is observed in the psoriatic lesions: TNF- $\alpha$, IFN- $\gamma$, IL- 6, IL- 8 , IL-12, IL-17, IL-18 along with the decreased concentration of anti-inflammatory cytokines: IL-10 and IL-4. Three cytokines, namely TNF- $\alpha, \mathrm{IL}-17$ and IFN- $\gamma$, seem to play the key role in the development of psoriatic lesions. Subcutaneous IFN- $\gamma$ administration induces the formation of psoriatic lesions. Interleukin-17 and IFN- $\gamma$ synergistically stimulate keratinocytes to synthesize IL-6, IL-7, IL-8, IL-12, IL-15, IL-18 and TNF- $\alpha$. Based on the current knowledge, TNF and IFN- $\gamma$ are called the primary inflammatory factors, as they induce the synthesis of secondary inflammatory cytokines and chemokines [12-15].

It has been confirmed that in psoriasis the recruitment of leukocytes to the skin is mediated by the chemokine and chemotactic cytokine network, of which the most important are IL-8 (CXCL8), fractalkine (CX3CL1), CCL5 (RANTES), CCL2 (MCP-1), CCL20 (MIP-3 $\alpha$ ), CCL26 (eotaxin2), CCL-27 (CTACK), and CXCL10 (Table 1).

Nomura et al. [16] compared gene expression RNA profiles of the skin lesions in atopic dermatitis and psoriasis using the genomic microarray techniques. A significantly higher expression of the following cytokines was reported for psoriatic changes: CCL4 (MIP-1 $\beta$ ), CCL20 (MIP-3 $\alpha$ ), CXCL8 (IL-8) and CXCL2 (GRO- $\beta$ ) CXCR2/IL-8R, while in atopic dermatitis CCL-13/MCP-4, CCL-18/PARC and CCL-27/CTACK [16] showed a higher expression.

What is the significance of an increased chemokine expression in the pathogenesis of psoriasis? It is recognized that CCL4 chemokine is a chemoattractant for cells bearing CCR1 and CCR5 receptors, i.e. Th1 cells, immature dendritic cells, NK cells and monocytes. CCL2O attracts cells with CCR6 receptor - Th1 lymphocytes, dendritic cells and monocytes, and CXCL8 and CXCL2 are chemoattractants for neutrophils expressing receptors for IL-8 
Table 1. Chemokines which expression increases in psoriatic lesions [16, 17]

\begin{tabular}{lcl}
\hline Chemokine & Receptor & Role in psoriasis pathogenesis \\
\hline CCL2/MCP-1 & CCR2 & DC and LC chemotaxis to the skin \\
\hline CCL4/MIP-1 $\beta$ & CCR1, CCR5 & Th1 lymphocyte, DC and monocyte chemotaxis to the skin \\
\hline CCL19/MIP-3 $\beta$ & CCR7 & T lymphocyte and DC chemotaxis to the lymph nodes \\
\hline CCL20/MIP-3 $\alpha$ & CCR6 & DC and LC chemotaxis to the skin \\
\hline CCL21/SLC & CCR7 & T lymphocyte and DC chemotaxis to the lymph nodes \\
\hline CCL18/PARC & Unknown & CD4 and CD8 lymphocyte chemotaxis \\
\hline CCL27/CTACK & CCR10 & T CLA+ lymphocyte chemotaxis \\
\hline CXCL5/ENA78 & CXCR2 & Neutrophil chemotaxis to the skin \\
\hline CXCL8/IL-8 & CXCR1, CXCR2 & As above \\
\hline CXCL1/GRO- $\alpha$ & CXCR2 & As above \\
\hline CXCL9/MIG & CXCR3 & T lymphocyte chemotaxis \\
\hline CXCL12/SDF-1 & CXCR4 & Neutrophil, monocyte, lymphocyte and DC chemotaxis \\
\hline CX3CL1 ( fractalkine) & CXCR1 & T lymphocyte chemotaxis to the skin \\
\hline
\end{tabular}

$D C$ - dendritic cell, $L C$ - Langerhans cell

(CXCR1 and CXCR2). In this context, it is very interesting that a new genetic phenomenon was reported in patients with psoriasis: multiplication of CCL4 gene copy number, which correlated with increased levels of CCL4 in the serum [17].

It was also demonstrated that one of the well-known "psoriasis susceptibility genes", with chromosome 3 locus, encodes a chemokine receptor - CX3CR1, which ligand - CX3CL1 (fractalkine) shows a high expression in psoriatic plaques [18]. Their interaction causes the trafficking of CX3CR1+ T lymphocytes to the inflamed skin. Another interesting discovery was finding, in an animal model, that fractalkine is one of the main chemotactic factors for immature mast cells, as an increased inflow of mast cells is observed in the skin in patients with psoriasis. Th1 CXCR3+ CCR5+ lymphocytes chemotaxis, reported in psoriasis, is also mediated by the increased expression of chemokines such as CXCL10 and CCL5 in the psoriatic skin [19].

Interleukin-8, i.e. CXCL8 chemokine (inflammation mediator synthesized de novo by mast cells) is the best described chemokine/cytokine in the context of psoriasis. It is a chemoattractant for neutrophils, which role in psoriasis is still not fully understood. Attempts to implement CXCL8 neutralizing therapy (ABX-IL8) were made, however were disappointing. Poor clinical efficacy could probably be attributed to IL-8/CXCL8 binding to two other receptors: CXCR1 and CXCR2 [9], which in turn bind also with other cytokines, especially with CXCL1-3 and CXCL57, which by interaction with CXCR1 and CXCR2 lead to "by-passing" CXCL8 pathway blocked by the drug $[9,10]$.
Recent studies have demonstrated, in both psoriasis and atopic dermatitis, the presence of CCL27/CTACK chemokine, produced only by keratinocytes and binding to the CCR10 receptor, characteristic of the majority of T lymphocytes infiltrating the skin [20-22]. CCL27 chemokine in responsible for lymphocyte adhesion to the skin [23]. CCL27 secretion is stimulated by TNF- $\alpha$, while corticosteroids and anti-TNF- $\alpha$ drugs increasingly used in the treatment of severe psoriasis (the so-called biologicals), reduce both the expression and function of CCL27. This stands as an indirect confirmation of the CCL27 role in the induction of the disease process in psoriasis [24-26].

CCR4 plays a role in the recruitment of the memory T lymphocyte to the skin together with CCR10, that is present on memory cells. CCR4 is accompanied by CCL17, a chemokine secreted by the endothelial cells of the skin blood vessels [27, 28]. CCR4 and CXCR3 receptors are highly expressed on the leukocytes which infiltrate the skin lesions, while in the dermis a high expression of their ligands is found: CCL17 and CCL22 together with CXCL9 and CXCL10. Epidermis in psoriatic lesions does not express CCR4, while CXCR3 is expressed. There are molecules (for example, T487) that bind to CXCR3 and inhibit the migration of lymphocytes into the inflamed tissue (skin in psoriasis, joints in rheumatoid arthritis, or gut in the autoinflammatory diseases) [29, 30]. It is also known that the antibody used in psoriasis treatment which neutralize p40 domain of IL-23 domain reduces the levels of CXCL8, CCL2 and CXCL10 in psoriatic lesions, leading to an effective reduction in disease activity index (PASI) [31]. 
CCL2O (MIP-3 $\alpha)$ is another chemokine that plays an important role in the pathogenesis of psoriasis. It is secreted by keratinocytes and acts as a chemoattractant for T lymphocytes and dendritic cells. CCL20 interacts with CCR6 receptor, highly expressed in psoriatic lesions, especially on $T$ cells with skin populating antigen - CLA+ [32-34]. It is suggested that the presence of mast cells in psoriatic lesions is associated with the overexpression of antimicrobial peptides hBD2 and cathelicidin LL37 in them [19].

\section{Cytokines and chemokines in atopic dermatitis}

Atopic dermatitis (AD), also known as atopic eczema, is a chronic, relapsing inflammatory disease of the skin. It affects $10-20 \%$ of children and $3 \%$ of adults. Atopic dermatitis is the most common allergic skin disease. Pathogenesis of $A D$ is complex and still not fully understood. The inflammatory infiltrates contain CD4+ lymphocytes expressing skin colonisation antigen (CLA), eosinophils, histiocytes, mast cells and Langerhans cells. Two phases can be distinguished in the course of AD: acute and chronic. Acute phase is characterized by the increase in the number of TNF- $\alpha$ secreting Langerhans cells with the increased surface expression of FceRI receptor with high IgE affinity. These LC are also called inflammatory dendritic cells (IDEC). An increased IgE production by $B$ lymphocytes and the influx of Th-2 lymphocytes, secreting IL-4, IL-5 and IL-13 into the skin is also observed in the acute phase. Chronic phase is, on the other hand, characterized by activation of Th-1 lymphocytes which produce mainly IFN- $\gamma$, TNF- $\alpha$, IL-8 and IL-12. It has been demonstrated that the reduced production of TNF- $\alpha$ and IFN- $\gamma$ together with increased IL-4 and IL-13 production may result in an increased susceptibility of the skin to Staphylococcus aureus infections well documented in AD patients [35].

Interleukin-18 plays a separate pleiotropic role in the cytokine network in AD pathogenesis. Interleukin-18 controls the balance between Th1 and Th2 response. Interleukin-18, synergistically with IL-12, promotes Th-1 response, increasing the synthesis of IFN- $\gamma$ and inhibiting the production of IgE antibodies by B lymphocytes. On the other hand, IL-18 increases the synthesis of IL-4 and $\mathrm{IL}-13$, promoting the synthesis of IgE. In the presence of IL-3 it stimulates the basophils and mast cells to secrete cytokines, chemokines and other mediators produced and stored in these cells [36].

Interleukin-31 is one of the mediators of pruritus in AD [37]. Interleukin-31 also affects the expression of chemokines. Interleukin-31 addition to cultured keratinocytes induces an increase in CCL1, CCL17 and CCL22 expression [38]. In addition to histamine and neuropeptides, mast cells which store tryptase and chymase in their cytoplasm and which directly act on C nerve fibers also participate in the origin of the itching sensation [39]. The abovementioned chemokines mediate pruritus, which does not respond fully to antihistaminic treatment.

Table 2. Chemokines and their role in atopic dermatitis pathogenesis

\begin{tabular}{|c|c|c|c|}
\hline Chemokine & Secreting cells & Receptor & Target cell \\
\hline CCL1/I-309 & $\begin{array}{l}\text { Keratinocytes, epithelial cells, } \\
\text { mast cells }\end{array}$ & CCR8 & T lymphocytes, LC precursors \\
\hline CCL2/MCP-1 & Keratinocytes, leukocytes & CCR2 & Monocytes, DC precursors \\
\hline CCL3/MIP-1 $\alpha$ & Leukocytes & CCR1, CCR5 & Monocytes, T lymphocytes \\
\hline CCL4/MIP-1 $\beta$ & Leukocytes & CCR5 & Monocytes, T lymphocytes \\
\hline CCL5/RANTES & Keratinocytes, leukocytes & CCR1, CCR3, CCR5 & Eosinophils, T lymphocytes, fibroblasts \\
\hline CCL11/eotaxin & Keratinocytes, T lymphocytes & CCR3 & Eosinophils, T lymphocytes \\
\hline CCL13/MCP-4 & $\begin{array}{l}\text { Keratinocytes, macrophages } T \\
\text { lymphocytes }\end{array}$ & CCR2, CCR3 & Monocytes, DC precursors, eosinophils, T lymphocytes \\
\hline CCL17/TARC & Keratinocytes, endothelial cells & CCR4 & T lymphocytes \\
\hline CCL18/PARC & Keratinocytes, DC & Unknown & T lymphocytes \\
\hline CCL20/MIP-3 $\alpha$ & Keratinocytes & CCR6 & T lymphocytes, monocytes, immature DC, LC \\
\hline $\mathrm{CCL} 22 / \mathrm{MDC}$ & Macrophages, DC & CCR4 & T lymphocytes \\
\hline CCL26/eotaxin-3 & Keratinocytes, endothelial cells & CCR3 & Eosinophils, T lymphocytes \\
\hline CCL27/CTACK & Keratinocytes & CCR10 & T lymphocytes \\
\hline $\mathrm{CX}_{3} \mathrm{CL} 1 /$ fractalkine & Endothelial cells & CX3CR1 & T lymphocytes, NK, monocytes, DC \\
\hline
\end{tabular}


Many chemokines and their receptors are involved in the pathogenesis of AD (Table 2). The complex chemokine web affects cell trafficking to the skin and formation of the inflammatory infiltrate involving $T$ lymphocytes, dendritic cells, eosinophils, histiocytes, and mast cells. The following chemokines play a crucial role in lymphocyte chemotaxis: CCL17 (TARC), CCL22 (MDC), CCL27 (CTACK), CCL18 (PARC) and IL-18. CCL20 (MIP-3 $\alpha$ ), CCL5 (RANTES), CCL2 (MCP-1) affect the migration of the dendritic cells. CCL11 (eotaxin), CCL13 (MCP-4) and CCL26 (eotaxin 3) act as chemotactic factors for eosinophils [40].

Chemokines and their receptors play an important role in $A D$ orchestrating the initiation and the exacerbation of the inflammation in response to allergens. Interleukin- 1 and TNF- $\alpha$ enhance CCL27 production by keratinocytes. CCL27 affects the process of T CLA + CCR10 + and/or CCR4 + lymphocytes recruitment to the skin [40, 41]. CCL17 - CCR4 ligand, secreted by the vascular endothelial cells of the skin and other tissues, supports the migration of CLA+ T cells towards the skin. These lymphocytes, activated in the skin by allergens or superantigens, consequently secrete effector cytokines such as IL-4, IL-13 and IFN- $\gamma$. These, in turn, exacerbate the production of CCL11, CCL26 by the target cells (keratinocytes, endothelial cells and T cells), affecting the migration of eosinophils to the skin. Th2 cytokines inhibit the production of antimicrobial peptides, thereby facilitating colonization of the skin by Staphylococcus aureus. The bacteria stimulate the production of CCL1 and CCL18 (in dendritic cells, keratinocytes, and endothelial cells), which facilitate migration of CLA+ T cells and Langerhans cells to the skin. On top of it, IgE-allergen complexes intensify CCL1 production in mast cells. CCR2 and CCR6 ligands (CCL2 and CCL20, respectively) also enhance the recruitment of dendritic cells to the atopic skin. These cells mature under the influence of pro-inflammatory cytokines, and begin to show the expression of CCR7. Due to the response to CCL21 ligand, they migrate to local lymph nodes, where they present antigenic determinants to $T$ lymphocytes. As a consequence, $T$ cells differentiate and express tissue-specific receptors and are recirculated to the areas of the allergen exposure in the human body $[40,41]$. This process is gradual (acute phase $\rightarrow$ chronic phase) and varies depending on the patient age. Notably low levels of CCL-17, CXCL-9, CXCL-10, IL-18, and high levels of CXCL-12, and CCL-27 were found in the in the blood of $A D$ patients younger than 10 years of age (lower than in the healthy control group). High levels of CCL-17, CCL22, CXCL-12 were demonstrated in the blood of older AD patients (> 10 years of age). A correlation of CCL-27 with eosinophilia and a correlation of CCL-17, CCL-27 and IL-18 with a total IgE level was also found in the latter group [42]. These differences make it necessary to review the approach to AD studies and include amongst other an age criterion.

\section{Cytokines and chemokines in mastocytosis}

The profile of chemokines and their receptors in mastocytosis is the least known. Mastocytosis is a heterogeneous group of rare diseases characterized by pathological accumulation of mast cells (MC). The disease usually involves the skin (cutaneous mastocytosis - CM), rarely the internal organs are affected, but of those the bone marrow, liver, spleen and lymph nodes are most often affected (systemic mastocytosis - SM). The pathophysiology of the disease is not yet completely understood. It is believed the mechanisms responsible for the proliferation and maturation of MC play a role in its pathogenesis. Activating mutation of the c-kit oncogene, encoding the stem cell factor (SCF) receptor, is considered to be the main mastocytosis inducing factor. In cutaneous mastocytosis the etiopathomechanism is not completely understood, as the factors responsible for MC proliferation and overactivity are not yet determined. Growth factors, cytokines and chemokines that stimulate/inhibit the proliferation of mast cells and their migration to the tissues produced by MC themselves and by epithelial cells are believed to be of importance [43].

Literature lacks publications on chemokines and their receptors expression in various clinical forms of mastocytosis. Only results obtained from a mast cell leukemic cell line HMC-1 are available. HMC-1 cells are a source of many chemokines: CCL1, CCL2, CCL3, CCl4, CCL5 (RANTES) and CXCL8 (IL-8), but, in contrast to other chemokines, CCL2 expression was significantly inhibited by glucocorticoids [41]. Leukemic mast cells express CXCR1 and CXCR2 receptors, both accepting IL-8 as a ligand. HMC- 1 cells migrate in response to GRO- $\alpha$, NAP-2, IP-10, complement components: C $3 \mathrm{a}, \mathrm{C} 5 \mathrm{a}$ and in response to TNF- $\alpha$. Experimental models showed the following to be the most important chemoattractants for mast cells: TNF- $\alpha$, TGF- $\beta 1$, VEGF, PGDF, SCF, as well as RANTES, NGF and complement components (C3a, $\mathrm{C} 5 \mathrm{a}, \mathrm{C} 1 \mathrm{q})$. According to some authors, chemotactic factors for mast cell cytokines may also include: IL-3, IL-4, IL-6, IL-15, IL-16 and IL-18, defensins: LL-37, HhBD-2, histamine and leukotrienes [19, 43-45].

\section{Summary}

Chemokines and cytokines are signaling peptides secreted by the resident skin cells as well as the cells forming the inflammatory infiltrates. They act in a paracrine manner - on the neighbor cells or in an autocrine way - on the secreting cell itself. Their primary role is to regulate the trafficking of white blood cells in order to maintain the proper function of the immune system and combat pathogens. They are also involved in neoangiogenesis, embryogenesis and organogenesis. Excessive activation as well as cytokine and chemokine synthesis are the hallmark of pathogenesis of many diseases: autoimmune (rheumatoid arthritis, psoriasis), inflammato- 
ry (atopic dermatitis, allergies, asthma, atherosclerosis), and those on the border of neoplasms as mastocytosis. That is why chemokines, cytokines and their receptors have revealed as new promising therapy targets in many chronic dermatoses.

\section{Acknowledgments}

The contribution of the first two authors was equal.

\section{Conflict of interest}

All authors declare no conflict of interest.

\section{References}

1. Gołąb J, Jakóbisiak M, Lasek W. Immunology. PWN Warsaw 2002.

2. Borish LC, Steinke JW. Cytokines and chemokines. J Allergy Clin Immunol 2003; 111 (2 Suppl): S460-75.

3. Baggiolini M. Chemokines in pathology and medicine. J Int Med 2001; 250: 91-104.

4. Gerard C, Rollins BJ. Chemokines and disease. Nature Immunol 2001; 2: 108-15.

5. Charo IF, Ransohoff RM. The many roles of chemokines and chemokine receptors in inflammation. N Engl J Med 2006; 354: 610-21.

6. Mabuchi T, Chang TW, Quinter S, et al. Chemokine receptors in the pathogenesis and therapy of psoriasis. I Dermatol Sci 2012; 65: 4-11.

7. Sokołowska-Wojdyło M, Roszkiewicz J. Role of the chemokines and their receptors in cutenaous T cell lymphomas [Polish]. Przegl Dermatol 2004; 91: 509-15.

8. Mazur G, Jaskuła E, Kryczek I. Role of chemokines in neoplasic diseases [Polish]. Adv Clin Exp Med 2004; 13: 315-25.

9. Zlotnik A, Yoshie O. Chemokines: a new classification system and their role in immunity. Immunity 2000; 12: 121-7.

10. Zlotnik A, Morales J, Hedrick JA. Recent advances in chemokines and chemokine receptors. Crit Rev Immunol 1999; 19: $1-47$.

11. Nedoszytko B, Roszkiewicz J. The role of the subpopulations of dendritic cells in psoriasis [Polish]. Postep Derm Alergo 2007; 24: 263-70.

12. Shaw FL, Kimber I, Begum R, et al. No impairment of monocyte-derived Langerhans cell phenotype or function in early-onset psoriasis. Clin Exp Dermatol 2012; 37: 40-7.

13. Krueger JG, Bowcock A. Psoriasis pathophysiology: current concepts of pathogenesis. Ann Rheum Dis 2005; 64 Suppl. 2: ii30-6.

14. Krueger JG, Ellis CN. Psoriasis - recent advances in understanding its pathogenesis and treatment. J Am Acad Dermatol 2005; 53 (1 Suppl. 1): S94-100.

15. Nedoszytko B. The role of subpopulations of lymphocytes in psoriasis [Polish]. Postep Derm Alergol 2008; 25: 20-33.

16. Nomura I, Gao B, Boguniewicz M, et al. Distinct patterns of gene expression in the skin lesions of atopic dermatitis and psoriasis: a gene microarray analysis. J Allergy Clin Immunol 2003; 112: 1195-202.

17. Zhou X, Krueger JG, Kao MC, et al. Novel mechanisms of T-cell and dendritic cell activation revealed by profiling of psoriasis on the 63,100-element oligonucleotide array. Physiol Genomics 2003; 13: 69-78.
18. Raychaudhuri SP, Jiang WY, Farber EM. Cellular localization of fractalkine at sites of inflammation: antigen-presenting cells in psoriasis express high levels of fractalkine. Br J Dermatol 2001; 144: 1105-13.

19. Aung G, Niyonsaba F, Ushio H, et al. Catestatin, a neuroendocrine antimicrobial peptide, induces human mast cell migration, degranulation and production of cytokines and chemokines. Immunology 2011; 132: 527-39.

20. Homey B, Alenius H, Müller A, et al. CCL27-CCR10 interactions regulate T cell-mediated skin inflammation. Nat Med 2002; 8: 157-65.

21. Homey B, Wang W, Soto $\mathrm{H}$, et al. Cutting edge: the orphan chemokine receptor $\mathrm{G}$ protein-coupled receptor-2 (GPR-2, CCR10) binds the skin-associated chemokine CCL27 (CTACK/ ALP/ILC). J Immunol 2000; 164: 3465-70.

22. Morales J, Homey B, Vicari AP, et al. CTACK, a skin-associated chemokine that preferentially attracts skin-homing memory T cells. Proc Natl Acad Sci U S A 1999; 96: 14470-5.

23. Plant $D$, Young HS, Watson R, et al. The CX3CL1-CX3CR1 system and psoriasis. Exp Dermatol 2006; 15: 900-3.

24. Gottlieb AB, Masud S, Ramamurthi R, et al. Pharmacodynamic and pharmacokinetic response to anti-tumor necrosis factor-alpha monoclonal antibody (infliximab) treatment of moderate to severe psoriasis vulgaris. I Am Acad Dermatol 2003; 48: 68-75.

25. Kinney T, Rawlins M, Kozarek R, et al. Immunomodulators and "on demand" therapy with infliximab in Crohn's disease: clinical experience with 400 infusions. Am J Gastroenterol 2003; 98: 608-12.

26. Rau R. Adalimumab (a fully human anti-tumour necrosis factor alpha monoclonal antibody) in the treatment of active rheumatoid arthritis: the initial results of five trials. Ann Rheum Dis 2002; 61 Suppl. 2: ii70-3.

27. Reiss Y, Proudfoot AE, Power CA, et al. CC chemokine receptor (CCR) 4 and the CCR10 ligand cutaneous T cell-attracting chemokine (CTACK) in lymphocyte trafficking to inflamed skin. J Exp Med 2001; 194: 1541-7.

28. Campbell JJ, Haraldsen G, Pan J, et al. The chemokine receptor CCR4 in vascular recognition by cutaneous but not intestinal memory T cells. Nature 1999; 19: 776-80.

29. Rottman JB, Smith TL, Ganley KG, et al. Potential role of the chemokine receptors CXCR3, CCR4, and the integrin alpha Ebeta7 in the pathogenesis of psoriasis vulgaris. Lab Invest 2001; 81: 335-47.

30. Wells TN, Power CA, Shaw JP, et al. Chemokine blockers: therapeutics in the making? Trends Pharmacol Sci 2006; 27: 41-7.

31. Toichi E, Torres G, McCormick TS, et al. An anti-IL-12p40 antibody down-regulates type 1 cytokines, chemokines, and IL-12/IL-23 in psoriasis. J Immunol 2006; 177: 4917-26.

32. Homey B, Dieu-Nosjean MC, Wiesenborn A, et al. Up-regulation of macrophage inflammatory protein-3 alpha/CCL2O and CC chemokine receptor 6 in psoriasis. I Immunol 2000; 164: 6621-32.

33. Dieu-Nosjean MC, Massacrier C, Homey B, et al. Macrophage inflammatory protein 3alpha is expressed at inflamed epithelial surfaces and is the most potent chemokine known in attracting Langerhans cell precursors. J Exp Med 2000; 192: 705-18.

34. Koelink PJ, Overbeek SA, Braber S, et al. Targeting chemokine receptors in chronic inflammatory diseases: an extensive review. Pharmacol Ther 2012; 133: 1-18.

35. Leung DY, Bieber T. Atopic dermatitis. Lancet 2003; 361: 151-60. 
36. Trzeciak M, Sokołowska-Wojdyło M, Barańska-Rybak W, et al. Interleukin 18 - a pleiotropic cytokine involved in the Th1 and Th2 immunological response. Postep Derm Alergol 2011; 28: 309-12.

37. Siniewicz-Luzeńczyk K, Stańczyk-Przyłuska A, Zeman K. Correlation between serum interleukin 31 level and the severity of disease in children with atopic dermatitis. Postep Derm Alergol 2013; 30: 282-5.

38. Dillon SR, Sprecher C, Hammond A, et al. Interleukin 31, a cytokine produced by activated T cells, induces dermatitis in mice. Nat Immunol 2004; 5: 752-60. Erratum in: Nat Immunol 2005; 6: 114.

39. Steinhoff M, Neisius U, Ikoma A, et al. Proteinase-activated receptor-2 mediates itch: a novel pathway for pruritus in human skin. J Neurosci 2003; 23: 6176-80.

40. Pivarcsi A, Homey B. Chemokine network in atopic dermatitis: traffic signals of disease. Curr. Allergy Asthma Rep 2005; 5: 284-90.

41. Homey B, Steinhoff M, Ruzicka T, Leung DY. Cytokines and chemokines orchestrate atopic skin inflammation. J Allergy Clin Immunol 2006; 118: 178-89.

42. Narbutt J, Lesiak A, Sysa-Jedrzejowska A, et al. The imbalance in serum concentration of Th-1- and Th-2-derived chemokines as one of the factors involved in pathogenesis of atopic dermatitis. Mediators Inflamm 2009; 2009: 269541.

43. Lange M, Niedoszytko M, Nedoszytko B, et al. Aethiopathogenesis of mastocytosis: current options [Polish]. Postep Derm Alergol 2009; 26: 142-5.

44. Starska K, Brzezińska-Błaszczyk E. The role of stromal mast cells in the modification of CD4+CD25+Foxp3 regulatory $T$ cells, Th17 lymphocytes and cytotoxic lymphocytes Tc1 in the development and progression of tumor [Polish]. Post Hig Med Dośw 2010; 64: 408-16.

45. Theoharides TC, Alysandratos KD, Angelidou A, et al. Mast cells and inflammation. Biochim Biophys Acta 2012; 1822: 21-33. 